\title{
A New Generalisation of Sam-Solai's Multivariate Wigner Distribution of Kind-1of Type-A*
}

\author{
${ }^{1}$ Dr. G.S. David Sam Jayakumar, ${ }^{2}$ Dr.A.Solairaju, ${ }^{3}$ Mr.S.Dawood Ali \\ ${ }^{1,3}$ Assistant Professor,Jamal Institute of Management,Tiruchirappalli - 620020 South India. \\ ${ }^{2}$ Associate Professor, Dept. Of Mathematics, Jamal Mohamed college, Tiruchirappalli - 620020 South India.
}

\begin{abstract}
This paper proposed a new generalization of Sam-Solai's Multivariate Wigner distribution of Kind-1 of Type-A from the univariate case. Further, we find its Cumulation, Marginal, Conditional distributions, Generating functions and also discussed its special case. The special cases include the transformation of Samsolai's Multivariate Wigner distribution of Kind-1 of Type-A into Multivariate one parameter Wigner distribution of Kind-1 of Type-A, Multivariate Wigner distribution of Kind-1 of Type-B, Multivariate log-Wigner distribution of Kind-1 of Type-A and Multivariate Inverse -Wigner distribution of Kind-1 of Type-A. It is found that the conditional variance of Sam-Solai's Multivariate conditional Wigner distribution is heteroscedastic and the correlation was found to be -0.16. Area values of the bi-variate Wigner surface also extracted and Wigner surfaces, contours are also visualized.
\end{abstract}

Keywords: Sam-Solai's Multivariate Wigner distribution of Kind-1 of Type-A, Multivariate one parameter Wigner distribution of Kind-1 of Type-A, Multivariate Wigner distribution of Kind-1 of Type-B, Multivariate log-Wigner distribution of Kind-1 of Type-A and Multivariate Inverse -Wigner distribution of Kind-1 of Type-A

\section{Introduction}

The origin of the conical distributions was first studied by the famous Noble laureate Wigner by introducing the semi-circle law and its distribution in the statistical literature. In this modern scenario, the Wigner's semi-circle distribution was used in diverse fields. Many authors studied this distribution in Trigonometric perspective and some authors tried to extend the semi-circle law to the power law as well as to the higher dimensional circles such as spheres and hyper spheres. Wigner [1957] proposed the distribution of the roots of certain symmetric matrices and Berezin [1973] highlighted some remarks about the Wigner distribution with special reference to the semi-circular law. Moreover, Mardia [1975] studied the Von mises distribution function and Watson [1982, 1983] proposed some distributions on the circles and the spheres. Some authors studied the application of Wigner's semi-circle distribution such as Accardi et al [1996] and Hiafi [2000] with reference to the quantum electro dynamics and entropy functions respectively. On the other hand, Bai at al [1988], Fang at al [1990], Boutet de Monvel et al [1999] and Evans [2000] explored and studied the convergence law of Wigner's semicircle law, symmetric multivariate distributions which include circular distributions, distribution of large random matrices and von mises distribution for higher dimensions respectively. Similarly, shimizu et al [2002], Jones et al[2005] and Arthur pewsey[2007]studied and proposed the Pearson Type VII distribution with reference to spheres, explored a family of distributions on circle and highlighted the wrapped t-family of circular distributions respectively.Moreover,Soltani[2009] introduced the two-sided power distributions which includes wigner's sem-circle distribution is a special case and Toshihiro et al[2009] proposed the Sine skewed circular distributions. Finally, Arizmendi et al [2010] and shogo Kato et al [2010] investigated the classical G-type distribution and origin of the family of distributions on the circle arising from the Mobius transformations respectively. Based on the past reviews, the authors noted only little work is done in the multivariate generalization of the Wigner's semi-circle distribution. By utilizing this research gap, the authors proposed a new generalization of Multivariate Wigner distribution and its properties, form are discussed in the next section.

\section{II.Sam-Solai's Multivariate Wigner Distribution}

Definition 2.1: Let $X_{1}, X_{2}, X_{3}, \mathrm{~K} X_{q}$ are the random variables followed Continuous univariate Wigner semi-circle distribution with mean 0 and variance $R_{i}{ }^{2} / 4$ for all i ( $\mathrm{i}=1$ to $\mathrm{p}$ ). Then the density of Multivariate Sam-Solai's Wigner distribution of Kind-1 of Type-A is defined as

$$
\begin{gathered}
f\left(x_{1}, x_{2}, x_{3} \ldots, x_{p}\right)=\left\{\frac{16}{3 \pi}\left(\sum_{i=1}^{p} \sqrt{\frac{1}{2}\left(\frac{R_{i}+x_{i}}{R_{i}}\right)}\right)-(p-1)\right\}\left(\frac{3}{4}\right)^{p} \prod_{i=1}^{p} \frac{1}{R_{i}} \sqrt{\frac{1}{2}\left(\frac{R_{i}-x_{i}}{R_{i}}\right)} \\
\text { where } i^{1} j-R_{i} £ x_{i} £+R_{i} \quad R_{i}>0
\end{gathered}
$$


A New Generalisation Of Sam-Solai's Multivariate Wigner Distribution Of Kind-1of Type-A

Definition 2.2: -From (1) and if $Z_{i}=x_{i} / R_{i}$, then using multi-dimensional Jacobian of transformation, the Sam-Solai's multivariate standard Wigner distribution and its density is defined as

$$
\begin{gathered}
f\left(Z_{1}, Z_{2}, Z_{3} \ldots, Z_{p}\right)=\left\{\frac{16}{3 \pi}\left(\sum_{i=1}^{p} \sqrt{\frac{1+Z_{i}}{2}}\right)-(p-1)\right\}\left(\frac{3}{4}\right)^{p} \prod_{i=1}^{p} \sqrt{\frac{1-Z_{i}}{2}} \\
\text { where } i^{1} j-1 £ Z_{i} £+1
\end{gathered}
$$

Theorem 2.3: -The cumulative distribution function of the Sam-Solai's Multivariate Wigner distribution is defined by

$$
\begin{aligned}
& F\left(Z_{1}, Z_{2}, Z_{3} \ldots, Z_{p}\right)=\int_{-R_{1}-R_{2}}^{z_{1}} \int_{-R_{3}}^{z_{2}} \ldots \int_{-R_{p}}^{z_{3}}\left\{\frac{16}{3 \pi}\left(\sum_{i=1}^{p} \sqrt{\left.\frac{1}{2}\left(\frac{R_{i}+u_{i}}{R_{i}}\right)\right)}-(p-1)\right\}\left(\frac{3}{4}\right)^{p} \prod_{i=1}^{p} \frac{1}{R_{i}} \sqrt{\frac{1}{2}\left(\frac{R_{i}-u_{i}}{R_{i}}\right)} d u_{i}\right. \\
& \text { where } i^{1} j-R_{i} £ u_{i} £ Z_{i} \\
& F\left(Z_{1}, Z_{2}, Z_{3} \mathrm{~K}, Z_{p}\right)=\left(\frac{1}{4}\right)^{p} \stackrel{p}{\mathrm{O}} \frac{1}{R_{i}^{3 / 2}}\left(4 R_{i}^{3 / 2}-\sqrt{2}\left(R_{i}-Z_{i}\right)^{3 / 2}\right)\left\{\left(\stackrel{\stackrel{p}{i}}{i=1}_{\frac{p}{2}+\frac{Z_{i} \sqrt{\left(R_{i}^{2}-Z_{i}^{2}\right)}}{p R_{i}^{2}}+\frac{\arcsin \left(Z_{i} / R_{i}\right)}{p}}^{\frac{1}{4 R_{i}^{3 / 2}}\left(4 R_{i}^{3 / 2}-\sqrt{2}\left(R_{i}-Z_{i}\right)^{3 / 2}\right)}\right)-(p-1)\right\}
\end{aligned}
$$

Theorem2.4: -The cumulative distribution function of the Sam-Solai's Multivariate standard Wigner distribution is defined by

$$
\begin{gathered}
F\left(Z_{1}, Z_{2}, Z_{3} \ldots, Z_{p}\right)=\int_{-1}^{z_{1}} \int_{-1}^{z_{2}} \int_{-1}^{z_{3}} \ldots \int_{-1}^{z_{p}}\left\{\frac{16}{3 \pi}\left(\sum_{i=1}^{p} \sqrt{\frac{1+u_{i}}{2}}\right)-(p-1)\right\}\left(\frac{3}{4}\right)^{p} \prod_{i=1}^{p} \sqrt{\frac{1-u_{i}}{2}} d u_{i} \\
\text { where } i^{1} j-1 £ u_{i} £ Z_{i} \\
F\left(Z_{1}, Z_{2}, Z_{3} \mathrm{~K}, Z_{p}\right)=\underset{i=1}{\tilde{O}}\left(1-\frac{\sqrt{2}\left(1-Z_{i}\right)^{3 / 2}}{4}\right)\left\{\left(\stackrel{\mathrm{a}}{i=1}^{p} \frac{\frac{1}{2}+\frac{Z_{i} \sqrt{\left(1-Z_{i}^{2}\right)}+\arcsin \left(Z_{i}\right)}{p}}{\left(1-\frac{\sqrt{2}\left(1-Z_{i}\right)^{3 / 2}}{4}\right)}\right)-(p-1)\right\}
\end{gathered}
$$

Theorem 2.5: The Probability density function of Sam-Solai's Multivariate wigner distribution of $X_{1}$ on $X_{2}, X_{3}, \mathrm{~K} X_{P}$ is

$$
\begin{gathered}
f\left(x_{1} / x_{2}, x_{3} \ldots, x_{p}\right)=\frac{\left\{\frac{16}{3 \pi}\left(\sum_{i=1}^{p} \sqrt{\frac{1}{2}\left(\frac{R_{i}+x_{i}}{R_{i}}\right)}\right)-(p-1)\right\} \frac{3}{4 R_{1}} \sqrt{\frac{1}{2}\left(\frac{R_{1}-x_{1}}{R_{1}}\right)}}{\left\{\frac{16}{3 \pi}\left(\sum_{i=2}^{p} \sqrt{\frac{1}{2}\left(\frac{R_{i}+x_{i}}{R_{i}}\right)}\right)-(p-2)\right\}} \\
\text { where } i^{1} j-R_{1} £ x_{1} £+R_{1}-1 £ r_{i j} £+1
\end{gathered}
$$

Proof: It is obtained from

$$
f\left(x_{1} / x_{2}, x_{3} \mathrm{~K}, x_{p}\right)=\frac{f\left(x_{1}, x_{2}, x_{3} \mathrm{~K}, x_{p}\right)}{f\left(x_{2}, x_{3} \mathrm{~K}, x_{p}\right)}
$$

Theorem 2.6- Mean and Variance of Sam-Solai's Multivariate Conditional Wigner distribution are

$$
\begin{aligned}
E\left(x_{1} / x_{2}, x_{3} \ldots, x_{p}\right) & =\frac{-\frac{R_{1}}{5}\left\{\frac{16}{3 \pi}\left(\sum_{i=2}^{p} \sqrt{\frac{1}{2}\left(\frac{R_{i}+x_{i}}{R_{i}}\right)}\right)-(p-1)\right\}}{\left\{\frac{16}{3 \pi}\left(\sum_{i=2}^{p} \sqrt{\frac{1}{2}\left(\frac{R_{i}+x_{i}}{R_{i}}\right)}\right)-(p-2)\right\}} \\
V\left(x_{1} / x_{2}, x_{3} \ldots, x_{p}\right) & =E\left(x_{1}^{2} / x_{2}, x_{3} \ldots, x_{p}\right)-\left(E\left(x_{1} / x_{2}, x_{3} \ldots, x_{p}\right)\right)^{2} \\
\text { where } E\left(x_{1}^{2} / x_{2}, x_{3} \ldots, x_{p}\right) & =\frac{R_{1}^{2}\left(\frac{1}{4}+\frac{11}{35}\left\{\frac{16}{3 \pi}\left(\sum_{i=2}^{p} \sqrt{\frac{1}{2}\left(\frac{R_{i}+x_{i}}{R_{i}}\right)}\right)-(p-1)\right\}\right)}{\left\{\frac{16}{3 \pi}\left(\sum_{i=2}^{p} \sqrt{\frac{1}{2}\left(\frac{R_{i}+x_{i}}{R_{i}}\right)}\right)-(p-2)\right\}}
\end{aligned}
$$

Proof: The Mean and variance of Multivariate Conditional Wigner distribution are given as 


$$
\begin{aligned}
& E\left(x_{1} / x_{2}, x_{3} \mathrm{~K}, x_{p}\right)=\underset{-R_{1}}{\mathrm{O}} x_{1} f\left(x_{1} / x_{2}, x_{3} \mathrm{~K}, x_{p}\right) d x_{1} \\
& E\left(x_{1} / x_{2}, x_{3} \mathrm{~K}, x_{p}\right)=\underset{-R_{1}}{\stackrel{+}{+}} x_{1} \frac{\left\{\frac { 1 6 } { 3 p } \left(\stackrel{\mathrm{a}}{i=1}_{i=1}^{p} \sqrt{\left.\left.\frac{1}{2}\left(\frac{R_{i}+x_{i}}{R_{i}}\right)\right)-(p-1)\right\} \frac{3}{4 R_{1}} \sqrt{\frac{1}{2}\left(\frac{R_{1}-x_{1}}{R_{1}}\right)}}\right.\right.}{\left\{\frac{16}{3 p}\left(\stackrel{\mathrm{a}}{i=2}^{p} \sqrt{\frac{1}{2}\left(\frac{R_{i}+x_{i}}{R_{i}}\right)}\right)-(p-2)\right\}} d x_{1} \\
& E\left(x_{1} / x_{2}, x_{3} \ldots, x_{p}\right)=\frac{-\frac{R_{1}}{5}\left\{\frac{16}{3 \pi}\left(\sum_{i=2}^{p} \sqrt{\frac{1}{2}\left(\frac{R_{i}+x_{i}}{R_{i}}\right)}\right)-(p-1)\right\}}{\left\{\frac{16}{3 \pi}\left(\sum_{i=2}^{p} \sqrt{\frac{1}{2}\left(\frac{R_{i}+x_{i}}{R_{i}}\right)}\right)-(p-2)\right\}}
\end{aligned}
$$

Similarly

$$
\begin{aligned}
& E\left(x_{1}^{2} / x_{2}, x_{3} \mathrm{~K}, x_{p}\right)=\underset{-R_{1}}{\mathrm{O}} x_{1}^{2} \frac{\left\{\frac { 1 6 } { 3 p } \left(\mathrm{a}_{i=1}^{p} \sqrt{\left.\left.\frac{1}{2}\left(\frac{R_{i}+x_{i}}{R_{i}}\right)\right)-(p-1)\right\} \frac{3}{4 R_{1}} \sqrt{\frac{1}{2}\left(\frac{R_{1}-x_{1}}{R_{1}}\right)}}\right.\right.}{\left\{\frac{16}{3 p}\left(\stackrel{\mathrm{a}}{i=2}_{i=2}^{p} \sqrt{\frac{1}{2}\left(\frac{R_{i}+x_{i}}{R_{i}}\right)}\right)-(p-2)\right\}} d x_{1} \\
& E\left(x_{1}^{2} / x_{2}, x_{3} \ldots, x_{p}\right)=\frac{R_{1}^{2}\left(\frac{1}{4}+\frac{11}{35}\left\{\frac{16}{3 \pi}\left(\sum_{i=2}^{p} \sqrt{\frac{1}{2}\left(\frac{R_{i}+x_{i}}{R_{i}}\right)}\right)-(p-1)\right\}\right)}{\left\{\frac{16}{3 \pi}\left(\sum_{i=2}^{p} \sqrt{\frac{1}{2}\left(\frac{R_{i}+x_{i}}{R_{i}}\right)}\right)-(p-2)\right\}}
\end{aligned}
$$

Substitute (7) and (8) in (6) we obtained the Conditional variance of the Multivariate conditional Wigner distribution.

Theorem2.7-If there are $\boldsymbol{p}=(\boldsymbol{q}+\boldsymbol{k})$ random variables, such that $\boldsymbol{q}$ random variables $X_{1}, X_{2}, X_{3}, \mathrm{~K} X_{q}$ conditionally depends on the $\boldsymbol{k}$ variables $X_{q+1}, X_{q+2}, X_{q+3}, \mathrm{~K} X_{q+k}$, then the density function of Sam-Solai's multivariate conditional Wigner distribution is

$$
\begin{aligned}
& f\left(x_{1}, x_{2}, x_{3} \mathrm{~K}, x_{q} / x_{q+1}, x_{q+2}, x_{q+3} \mathrm{~K}, x_{q+k}\right)=\frac{\left\{\frac{16}{3 p}\left(\stackrel{\mathrm{a}}{\mathrm{a}}{ }_{i=1}^{q+k} \sqrt{\frac{1}{2}\left(\frac{R_{i}+x_{i}}{R_{i}}\right)}\right)-(q+k-1)\right\}\left(\frac{3}{4}\right)^{q} \stackrel{q}{\mathrm{O}} \frac{1}{R_{i}} \sqrt{\frac{1}{2}\left(\frac{R_{i}-x_{i}}{R_{i}}\right)}}{\left\{\frac{16}{3 p}\left(\mathrm{\stackrel {a } { a }}_{i=q+1}^{q+k} \sqrt{\frac{1}{2}\left(\frac{R_{i}+x_{i}}{R_{i}}\right)}\right)-(k-1)\right\}} \\
& \text { where } i^{1} \quad j-R_{i} £ x_{i} £+R_{i} \quad p=q+k
\end{aligned}
$$

Proof: Let the multivariate conditional law for $\boldsymbol{q}$ random variables $X_{1}, X_{2}, X_{3}, \mathrm{~K} X_{q}$ conditionally depends on the $\boldsymbol{k}$ variables $X_{q+1}, X_{q+2}, X_{q+3}, \mathrm{~K} X_{q+k}$ is given as

$$
\begin{aligned}
& f\left(x_{1}, x_{2}, x_{3}, \mathrm{~K} x_{q} / x_{q+1}, x_{q+2}, x_{q+3}, \mathrm{~K} x_{q+k}\right)=\frac{f\left(x_{1}, x_{2}, x_{3}, \mathrm{~K} x_{q}, x_{q+1}, x_{q+2}, x_{q+3}, \mathrm{~K} x_{q+k}\right)}{f\left(x_{q+1}, x_{q+2}, x_{q+3}, \mathrm{~K} x_{q+k}\right)}
\end{aligned}
$$

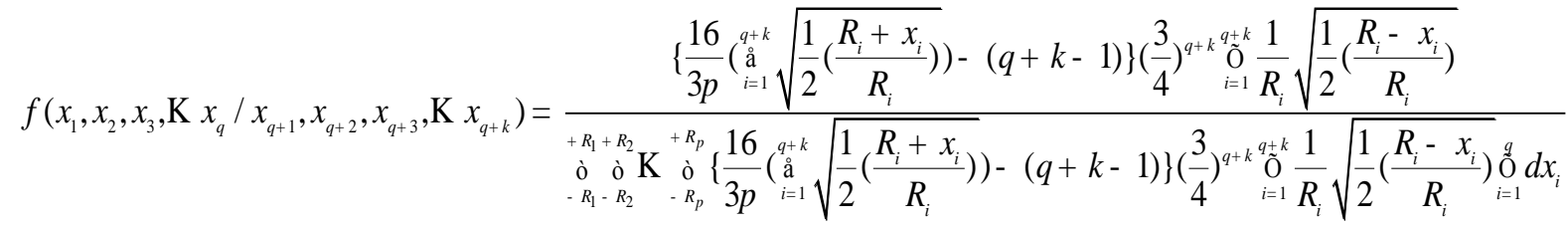

$$
\begin{aligned}
& f\left(x_{1}, x_{2}, x_{3} \mathrm{~K}, x_{q} / x_{q+1}, x_{q+2}, x_{q+3} \mathrm{~K}, x_{q+k}\right)=\frac{\left.\left\{\frac{16}{3 p} \stackrel{\mathrm{a}}{i=1}_{\mathrm{a}}^{q+k} \sqrt{\frac{1}{2}\left(\frac{R_{i}+x_{i}}{R_{i}}\right)}\right)-(q+k-1)\right\}\left(\frac{3}{4}\right)^{q} \mathrm{O}_{i=1}^{q} \frac{1}{R_{i}} \sqrt{\frac{1}{2}\left(\frac{R_{i}-x_{i}}{R_{i}}\right)}}{\left\{\frac{16}{3 p}\left(\mathrm{a}_{i=q+1}^{q+k} \sqrt{\frac{1}{2}\left(\frac{R_{i}+x_{i}}{R_{i}}\right)}\right)-(k-1)\right\}} \\
& \text { where } i^{1} \quad j-R_{i} £ x_{i} £+R_{i} \quad p=q+k
\end{aligned}
$$




\section{III.Constants of Sam-Solai's Multivariate Wigner Distribution}

Theorem 3.1 The Marginal Co-variance and Population Correlation Co-efficient between the random variables $X_{1}$ and $X_{2}$ is given as

$$
\operatorname{COV}\left(x_{1}, x_{2}\right)=-\frac{R_{1} R_{2}}{25}
$$

Proof: Let the product moment of the Sam-Solai's multivariate Wigner distribution in terms of Co-variance from the origin is given as

$$
\begin{aligned}
& \operatorname{COV}\left(x_{1}, x_{2}\right)=\underset{-R_{1}-R_{2}}{\mathbf{0}} \mathrm{O}_{-R_{p}}^{+R_{1}+R_{2}} \underset{\mathrm{O}}{+R_{p}} x_{1} x_{2} f\left(x_{1}, x_{2}, x_{3}, \mathrm{~K} x_{p}\right) \underset{i=1}{\stackrel{D}{O}} d x_{i}
\end{aligned}
$$

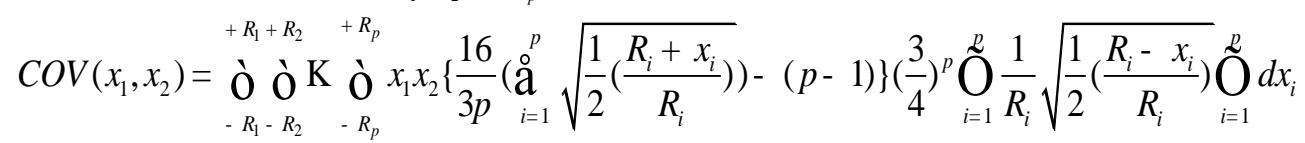

$$
\begin{aligned}
& \operatorname{COV}\left(x_{1}, x_{2}\right)=-R_{1} R_{2} / 25
\end{aligned}
$$

Correlation Co-efficient of a distribution is $\rho\left(x_{1}, x_{2}\right)=\operatorname{COV}\left(x_{1}, x_{2}\right) / \sigma_{1} \sigma_{2}$

It observes that $s_{1}=R_{1} / 2$ and $s_{2}=R_{2} / 2$

From (10), (11) and (12), it follows that $\rho\left(x_{1}, x_{2}\right)=-4 / 25$

Remark3.1: The result can be generalized to the Co-variance and Correlation between the $i^{\text {th }}$ and $j^{\text {th }}$ random variable are given as

$$
\begin{aligned}
& \operatorname{COV}\left(x_{i}, x_{j}\right)=-R_{i} R_{j} / 25 \\
& \rho\left(x_{i}, x_{j}\right)=-4 / 25 \quad \text { where } i^{1} j
\end{aligned}
$$

Theorem 3.2: The Moment generating function of Sam-Solai's Multivariate Wigner distribution is

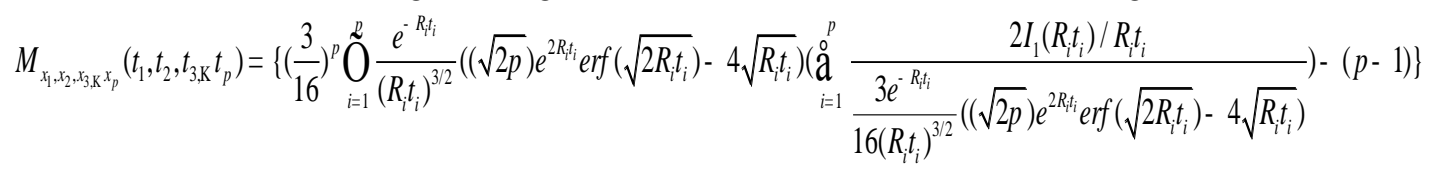

where $I_{1}\left(R_{i} t_{i}\right)$ is the modified Bessel function and $\operatorname{erf}\left(\sqrt{2 R_{i} t_{i}}\right)$ is the error function.

Proof: Let the moment generating function of the Multivariate distribution is given as

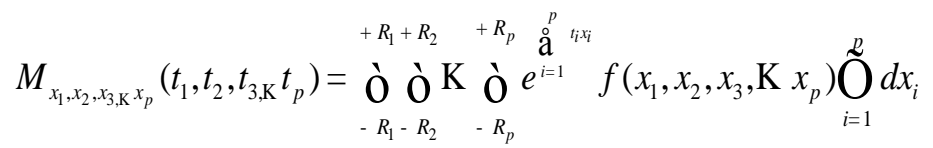

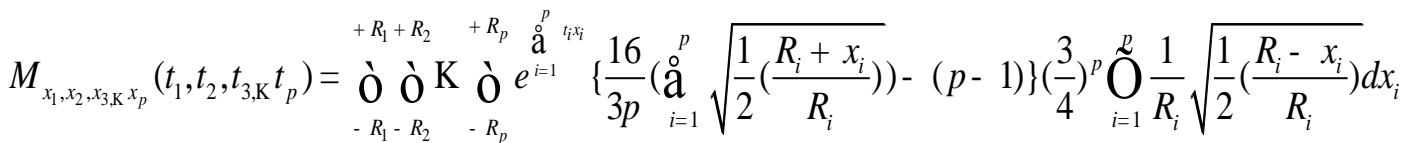

From (16), it observes that

$$
\begin{gathered}
\int_{-R_{i}}^{+R_{i}} e^{t_{i} x_{i}} \frac{2}{\pi R_{i}^{2}} \sqrt{R_{i}^{2}-x_{i}^{2}} d x_{i}=\frac{2 I_{1}\left(R_{i} t_{i}\right)}{R_{i} t_{i}} \\
\int_{-R_{i}}^{+R_{i}} e^{t_{i} x_{i}} \frac{3}{4 R_{i}} \sqrt{\frac{1}{2}\left(\frac{R_{i}-x_{i}}{R_{i}}\right)} d x_{i}=\frac{3 e^{-R_{i} t_{i}}}{16\left(R_{i} t_{i}\right)^{3 / 2}}\left((\sqrt{2 \pi}) e^{2 R_{i} t_{i}} \operatorname{erf}\left(\sqrt{2 R_{i} t_{i}}\right)-4 \sqrt{R_{i} t_{i}}\right) \\
\operatorname{erf}\left(\sqrt{2 R_{i} t_{i}}\right)=\frac{2}{\sqrt{\pi}} \int_{0}^{\sqrt{2 R_{i} t_{i}}} e^{-x_{i}^{2}} d x_{i}
\end{gathered}
$$

From (16), (17), (18) and (19), it follows that

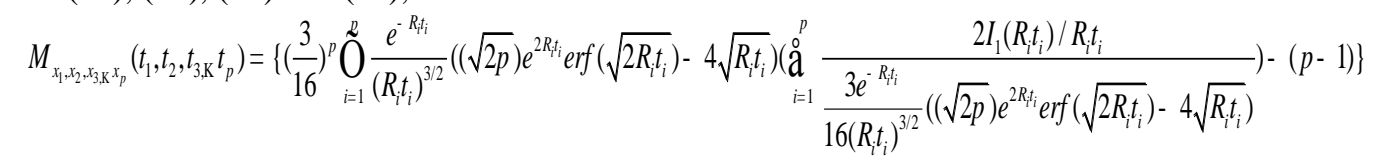

By integration. 
A New Generalisation Of Sam-Solai's Multivariate Wigner Distribution Of Kind-1of Type-A

Theorem 3.3: The Cumulant of the Moment generating function of the Sam-Solai's Multivariate Wigner distribution is

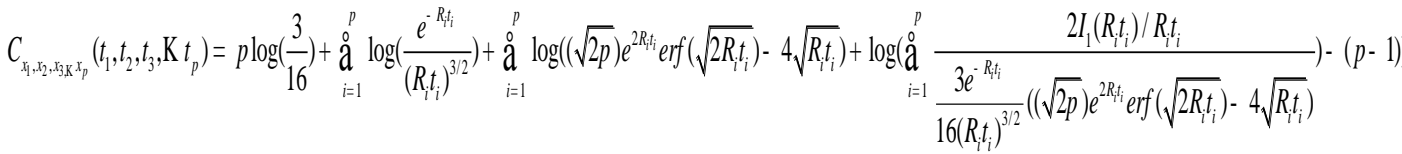

where $I_{1}\left(R_{i} t_{i}\right)$ is the modified Bessel function and $\operatorname{erf}\left(\sqrt{2 R_{i} t_{i}}\right)$ is the error function.

Proof: It is found from

$$
C_{x_{1}, x_{2}, x_{3, \mathrm{~K}} x_{p}}\left(t_{1}, t_{2}, t_{3, \mathrm{~K}} t_{p}\right)=\log \left(M_{x_{1}, x_{2}, x_{3 \mathrm{~K}} x_{p}}\left(t_{1}, t_{2}, t_{3, \mathrm{~K}} t_{p}\right)\right)
$$

Theorem 3.4: The Characteristic function of the Sam-Solai's Multivariate Wigner distribution is

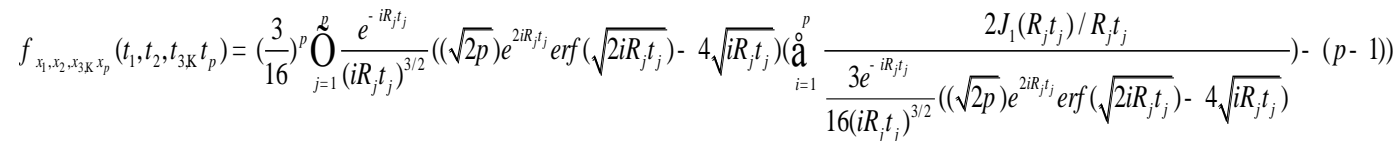

where $J_{1}\left(R_{j} t_{j}\right)$ the Bessel is function and $\operatorname{erf}\left(\sqrt{2 i R_{j} t_{j}}\right)$ is the complex error function.

Proof: Let the characteristic function of a multivariate distribution is given as

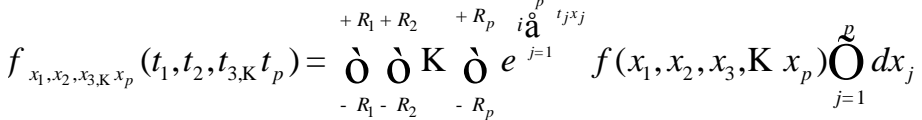

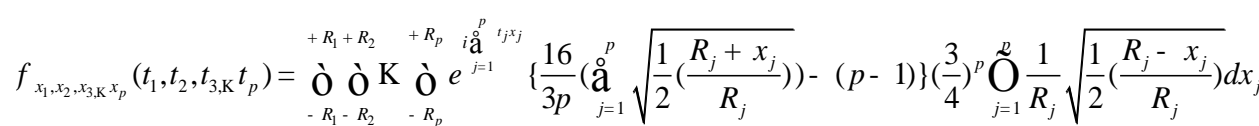

From (22), it observes that

$$
\begin{gathered}
\int_{-R_{j}}^{+R_{j}} e^{i t_{j} x_{j}} \frac{2}{\pi R_{j}^{2}} \sqrt{R_{j}^{2}-x_{j}^{2}} d x_{j}=\frac{2 J_{1}\left(R_{j} t_{j}\right)}{R_{j} t_{j}} \\
\int_{-R_{j}}^{+R_{j}} e^{i t_{j} x_{j}} \frac{3}{4 R_{j}} \sqrt{\frac{1}{2}\left(\frac{R_{j}-x_{j}}{R_{j}}\right)} d x_{j}=\frac{3 e^{-i R_{j} t_{j}}}{16\left(i R_{j} t_{j}\right)^{3 / 2}}\left((\sqrt{2 \pi}) e^{2 i R_{j} t_{j}} \operatorname{erf}\left(\sqrt{2 i R_{j} t_{j}}\right)-4 \sqrt{i R_{j} t_{j}}\right) \\
\operatorname{erf}\left(\sqrt{2 i R_{j} t_{j}}\right)=\frac{2}{\sqrt{\pi}} \int_{0}^{\sqrt{2 i R_{j} t_{j}}} e^{-x_{j}^{2}} d x_{j}
\end{gathered}
$$

From (22), (23), (24) and (25), it follows that

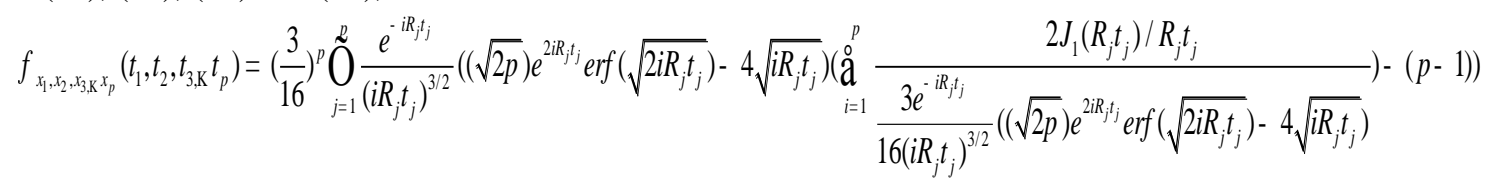

\section{IV.Some Special Cases}

by integration.

Result 4.1: From (1) and if $\boldsymbol{P}=\mathbf{1}$, then the Sam-Solai's multivariate Wigner density of Kind-1 of Type-A is reduced into product of the density function of uni-variate wigner semi-circle distributions.

Result 4.2: From (1) and if $\boldsymbol{P = 2}$, then the density of Sam-Solai's Multivariate Wigner distribution was reduced into

$$
\begin{gathered}
f\left(x_{1}, x_{2}\right)=\left\{\frac{16}{3 \pi} \sqrt{\frac{1}{2}\left(\frac{R_{1}+x_{1}}{R_{1}}\right)}+\frac{16}{3 \pi} \sqrt{\frac{1}{2}\left(\frac{R_{2}+x_{2}}{R_{2}}\right)}-1\right\} \frac{3}{4 R_{1}} \sqrt{\frac{1}{2}\left(\frac{R_{1}-x_{1}}{R_{1}}\right)} \frac{3}{4 R_{2}} \sqrt{\frac{1}{2}\left(\frac{R_{2}-x_{2}}{R_{2}}\right)} \\
\text { where }-R_{1} £ x_{1} £+R_{1}-R_{2} £ x_{2} £+R_{2} \quad R_{1}, R_{2}>0
\end{gathered}
$$

This is called Sam-Solai's Bi-variate Wigner distribution of Kind-1 of Type-A

Result 4.3: From (2) and if $\boldsymbol{P}=\mathbf{2}$, then the Sam-Solai's Bi-variate standard Wigner distribution and its density is given as

$$
\begin{gathered}
f\left(Z_{1}, Z_{2}\right)=\left\{\frac{16}{3 \pi} \sqrt{\frac{1+Z_{1}}{2}}+\frac{16}{3 \pi} \sqrt{\frac{1+Z_{2}}{2}}-1\right\}\left(\frac{3}{4} \sqrt{\frac{1-Z_{1}}{2}}\right)\left(\frac{3}{4} \sqrt{\frac{1-Z_{2}}{2}}\right) \\
\text { where }-1 £ Z_{1} £+1,-1 £ Z_{2} £+1
\end{gathered}
$$

This is called Sam-Solai's Bi-variate standard Wigner distribution of Kind-1 of Type-A 
Result 4.4: Below the diagram shows the Bi-variate probability surface and contour plot of the Sam-Solai's Bivariate standard Wigner distribution are given.

$-1 \leq Z_{1} \leq+1 \quad-1 \leq Z_{2} \leq+1 \quad \rho_{12}=-0.16$

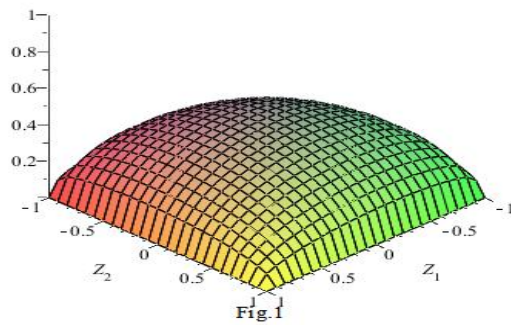

$-1 \leq Z_{1} \leq+1 \quad-1 \leq Z_{2} \leq+1 \quad \rho_{12}=-0.16$

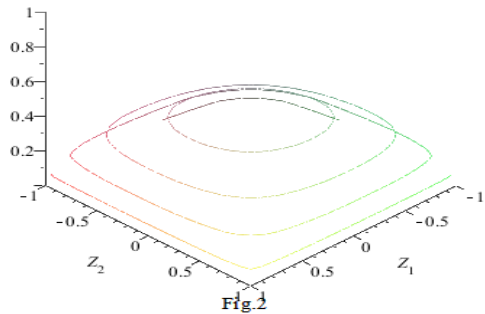

Result 4.5: From (4) and if $\boldsymbol{P = 2}$, then the Sam-Solai's Bi-variate Cumulative standard wigner distribution is given as

$$
F\left(Z_{1}, Z_{2}\right)=\left(1-\frac{\sqrt{2}\left(1-Z_{1}\right)^{3 / 2}}{4}\right)\left(1-\frac{\sqrt{2}\left(1-Z_{2}\right)^{3 / 2}}{4}\right)\left\{\frac{\frac{1}{2}+\frac{Z_{1} \sqrt{\left(1-Z_{1}^{2}\right)}+\arcsin \left(Z_{1}\right)}{p}}{\left(1-\frac{\sqrt{2}\left(1-Z_{1}\right)^{3 / 2}}{4}\right)}+\frac{\frac{1}{2}+\frac{Z_{2} \sqrt{\left(1-Z_{2}^{2}\right)}+\arcsin \left(Z_{2}\right)}{p}}{\left(1-\frac{\sqrt{2}\left(1-Z_{2}\right)^{3 / 2}}{4}\right)}-1\right\}
$$

Result 4.6: Below the diagram shows the Bi-variate cumulative probability surface and Cumulative Contour plot of the Sam-Solai's Bi-variate cumulative standard Wigner distribution are given.

$-1 \leq Z_{1} \leq+1 \quad-1 \leq Z_{2} \leq+1 \quad p_{12}=-0.16$

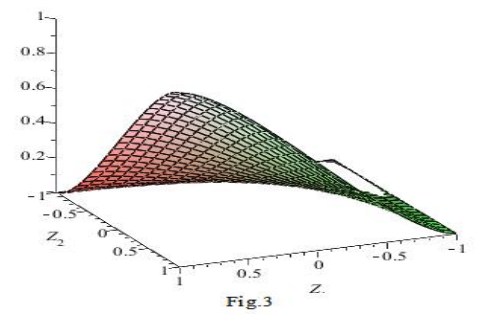

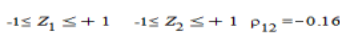

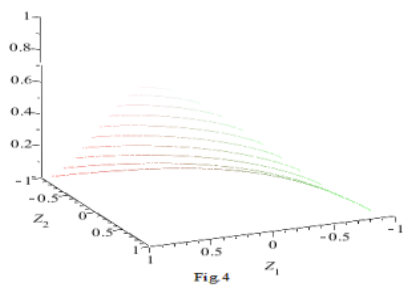

Result 4.7-Using the software Maple version 14, the table values from -0.9 to +0.9 with interval value 0.1 for Sam-Solai's bi-variate standard Wigner distribution are obtained. Area under the Sam-Solai's Bi-variate Wigner surfaces based on cumulative distribution function is given.

\begin{tabular}{|c|c|c|c|c|c|c|c|c|c|c|c|c|c|c|c|c|c|c|c|}
\hline \multirow{2}{*}{$Z_{1}$} & \multicolumn{19}{|c|}{$Z_{2}$} \\
\hline & -0.9 & -0.8 & -0.7 & -0.6 & -0.5 & -0.4 & -0.3 & -0.2 & -0.1 & $\theta$ & 0.1 & 0.2 & 0.3 & 0.4 & 0.5 & 0.6 & 0.7 & 0.8 & 0.9 \\
\hline a.9 & -0.003 & -0.006 & -0.008 & -0.011 & -0.013 & -0.015 & -0.017 & -0.018 & -0.019 & -0.02 & -0.02 & -0.02 & -0.02 & -0.019 & -0.017 & -0.015 & -0.012 & -0.007 & 0.000 \\
\hline-0.8 & -0.002 & -0.006 & -0.01 & -0.013 & -0.016 & -0.019 & -0.021 & -0.023 & -0.024 & -0.025 & -0.025 & -0.024 & -0.023 & -0.02 & -0.017 & -0.012 & -0.006 & 0.003 & 0.016 \\
\hline-2.7 & 0.001 & -0.002 & -0.006 & -0.009 & -0.012 & -0.015 & -0.017 & -0.018 & -0.019 & -0.019 & -0.018 & -0.016 & -0.014 & -0.01 & -0.004 & 0.003 & 0.012 & 0.025 & 0.044 \\
\hline-0.6 & 0.007 & 0.005 & 0.003 & 0.000 & -0.002 & -0.004 & -0.005 & -0.005 & -0.005 & -0.004 & -0.002 & 0.001 & 0.006 & 0.011 & 0.018 & 0.028 & 0.04 & 0.056 & 0.08 \\
\hline-0.5 & 0.0146 & 0.0158 & 0.0155 & 0.0149 & 0.0143 & 0.0141 & 0.0144 & 0.0153 & 0.017 & 0.0194 & 0.0229 & 0.0274 & 0.0333 & 0.0407 & 0.0499 & 0.0615 & 0.0761 & 0.0953 & 0.1227 \\
\hline-0.4 & 0.0246 & 0.0297 & 0.0323 & 0.0341 & 0.0358 & 0.0375 & 0.0397 & 0.0423 & 0.0457 & 0.0498 & 0.0549 & 0.0611 & 0.0687 & 0.0778 & 0.0889 & 0.1024 & 0.1193 & 0.141 & 0.1717 \\
\hline-0.3 & 0.0365 & 0.0464 & 0.0527 & 0.0576 & 0.0619 & 0.0661 & 0.0705 & 0.0753 & 0.0806 & 0.0866 & 0.0935 & 0.1015 & 0.1108 & 0.1217 & 0.1345 & 0.1498 & 0.1685 & 0.1924 & 0.2256 \\
\hline$-a .2$ & 0.0502 & 0.0657 & 0.0763 & 0.0848 & 0.0923 & 0.0994 & 0.1063 & 0.1135 & 0.121 & 0.1291 & 0.138 & 0.1478 & 0.1589 & 0.1714 & 0.1859 & 0.2028 & 0.2231 & 0.2486 & 0.2836 \\
\hline-0.1 & 0.0654 & 0.0873 & 0.1029 & 0.1155 & 0.1266 & 0.1368 & 0.1466 & 0.1564 & 0.1663 & 0.1766 & 0.1875 & 0.1993 & 0.2121 & 0.2262 & 0.2422 & 0.2605 & 0.2821 & 0.3087 & 0.3449 \\
\hline$\theta$ & 0.0821 & 0.1111 & 0.1321 & 0.1492 & 0.1642 & 0.1779 & 0.1909 & 0.2034 & 0.2159 & 0.2286 & 0.2416 & 0.2552 & 0.2698 & 0.2855 & 0.3028 & 0.3222 & 0.3447 & 0.3721 & 0.4087 \\
\hline 0.1 & 0.1001 & 0.1368 & 0.1636 & 0.1856 & 0.2048 & 0.2223 & 0.2385 & 0.2541 & 0.2692 & 0.2843 & 0.2994 & 0.315 & 0.3312 & 0.3483 & 0.3668 & 0.3872 & 0.4103 & 0.438 & 0.4743 \\
\hline$\overline{a .2}$ & 0.1192 & 0.164 & 0.1971 & 0.2243 & 0.248 & 0.2693 & 0.289 & 0.3077 & 0.3256 & 0.3431 & 0.3604 & 0.3778 & 0.3956 & 0.414 & 0.4335 & 0.4546 & 0.4781 & 0.5056 & 0.541 \\
\hline 0.3 & 0.1392 & 0.1926 & 0.2322 & 0.2648 & 0.2931 & 0.3185 & 0.3418 & 0.3636 & 0.3843 & 0.4043 & 0.4237 & 0.443 & 0.4623 & 0.4819 & 0.5022 & 0.5237 & 0.5472 & 0.5741 & 0.6081 \\
\hline 0.4 & 0.16 & 0.2222 & 0.2686 & 0.3068 & 0.3398 & 0.3694 & 0.3963 & 0.4213 & 0.4448 & 0.4672 & 0.4887 & 0.5097 & 0.5304 & 0.551 & 0.572 & 0.5937 & 0.6169 & 0.6428 & 0.6747 \\
\hline 0.5 & 0.1813 & 0.2526 & 0.3058 & 0.3496 & 0.3875 & 0.4212 & 0.4518 & 0.48 & 0.5062 & 0.531 & 0.5545 & 0.5771 & 0.599 & 0.6205 & 0.6419 & 0.6636 & 0.6861 & 0.7106 & 0.7399 \\
\hline 0.6 & 0.2028 & 0.2832 & 0.3433 & 0.3928 & 0.4355 & 0.4733 & 0.5075 & 0.5388 & 0.5677 & 0.5947 & 0.6201 & 0.6442 & 0.6672 & 0.6893 & 0.7109 & 0.7323 & 0.7539 & 0.7767 & 0.8028 \\
\hline 0.7 & 0.2242 & 0.3137 & 0.3806 & 0.4356 & 0.483 & 0.5249 & 0.5625 & 0.5967 & 0.6282 & 0.6573 & 0.6844 & 0.7097 & 0.7336 & 0.7562 & 0.7778 & 0.7986 & 0.8189 & 0.8395 & 0.8621 \\
\hline 0.8 & 0.2451 & 0.3434 & 0.4168 & 0.4772 & 0.5291 & 0.5747 & 0.6156 & 0.6525 & 0.6863 & 0.7172 & 0.7458 & 0.7722 & 0.7967 & 0.8195 & 0.8408 & 0.8607 & 0.8795 & 0.8976 & 0.9161 \\
\hline 0.9 & 0.265 & 0.3714 & 0.451 & 0.5162 & 0.5721 & 0.6212 & 0.6649 & 0.7043 & 0.7399 & 0.7724 & 0.8021 & 0.8293 & 0.8541 & 0.8768 & 0.8974 & 0.9162 & 0.9331 & 0.9484 & 0.9623 \\
\hline
\end{tabular}


Result 4.8: From (1) and if $R_{i}=R$, then the Sam-Solai's Multivariate wigner distribution of Kind-1 of Type-A transformed into Multivariate one parameter Wigner distribution of Kind-1 of Type-A and its density is given as

$$
\begin{gathered}
f\left(x_{1}, x_{2}, x_{3} \ldots, x_{p}\right)=\left\{\frac{16}{3 \pi}\left(\sum_{i=1}^{p} \sqrt{\left.\frac{1}{2}\left(\frac{R+x_{i}}{R}\right)\right)}-(p-1)\right\}\left(\frac{3}{4 R}\right)^{p} \prod_{i=1}^{p} \sqrt{\frac{1}{2}\left(\frac{R-x_{i}}{R}\right)}\right. \\
\text { where } i^{1} j-R £ x_{i} £+R \quad R>0
\end{gathered}
$$

Result 4.9: From (1) and if $y_{i}=-x_{i}$, then the Sam-Solai's Multivariate wigner distribution of Kind-1 of TypeA transformed into Sam-solai's Multivariate Wigner distribution of Kind-1 of Type-B and its density is given as

$$
\begin{gathered}
f\left(y_{1}, y_{2}, y_{3} \ldots, y_{p}\right)=\left\{\frac{16}{3 \pi}\left(\sum_{i=1}^{p} \sqrt{\frac{1}{2}\left(\frac{R_{i}-y_{i}}{R_{i}}\right)}\right)-(p-1)\right\}\left(\frac{3}{4}\right)^{p} \prod_{i=1}^{p} \frac{1}{R_{i}} \sqrt{\frac{1}{2}\left(\frac{R_{i}+y_{i}}{R_{i}}\right)} \\
\text { where } i^{1} j-R_{i} £ y_{i} £+R_{i} \quad R_{i}>0
\end{gathered}
$$

Result 4.10: From (1) and if $y_{i}=e^{x_{i}}$, then the Sam-Solai's Multivariate wigner distribution of Kind-1 of Type-A transformed into Sam-solai's Multivariate log- Wigner distribution of Kind-1 of Type-A and its density is given as

$$
\begin{gathered}
f\left(y_{1}, y_{2}, y_{3} \mathrm{~K}, y_{p}\right)=\left\{\frac{16}{3 p}\left(\stackrel{p}{\mathrm{a}} \sqrt{\frac{1}{2}\left(\frac{R_{i}+\log y_{i}}{R_{i}}\right)}\right)-(p-1)\right\}\left(\frac{3}{4}\right)^{p} \underset{i=1}{\stackrel{D}{\mathrm{O}}} \frac{1}{R_{i} y_{i}} \sqrt{\frac{1}{2}\left(\frac{R_{i}-\log y_{i}}{R_{i}}\right)} \\
\text { where } i^{1} j \quad e^{-R_{i}} £ y_{i} £ e^{R_{i}} \quad R_{i}>0
\end{gathered}
$$

Result 4.11: From (2) and if $y_{i}=1 / x_{i}$, then the Sam-Solai's Multivariate Wigner distribution of Kind-1 of Kind-1 of Type-A transformed into Sam-solai's Multivariate Inverse Wigner distribution of Kind-1 of Type-A and its density is given as

$$
\begin{gathered}
f\left(y_{1}, y_{2}, y_{3} \ldots, y_{p}\right)=\left\{\frac{16}{3 \pi}\left(\sum_{i=1}^{p} \sqrt{\frac{1}{2}\left(\frac{1+R_{i} y_{i}}{R_{i} y_{i}}\right)}\right)-(p-1)\right\}\left(\frac{3}{4}\right)^{p} \prod_{i=1}^{p} \frac{1}{R_{i} y_{i}^{2}} \sqrt{\frac{1}{2}\left(\frac{1-R_{i} y_{i}}{R_{i} y_{i}}\right)} \\
\text { where } i^{1} j-1 / R_{i} £ y_{i} £+1 / R_{i}, R_{i}>0
\end{gathered}
$$

\section{V.Conclusion}

The Sam-Solai's generalization of multivariate Wigner distribution of Kind-1 of Type-A is having some interesting features. At first, the marginal uni-variate distributions of the Sam-Solai's Multivariate Wigner distribution are uni-variate and enjoyed the symmetric property. Secondly, the standard correlation co-efficient between any two circular variables found to be -0.16 . Finally, the multivariate generalization of wigner's semicircle distribution of Kind- 1 of Type-A open the way for the transformation of the distribution into Multivariate one parameter Wigner distribution of Kind-1 of Type-A, Multivariate Wigner distribution of Kind-1 of Type-B, Multivariate log-Wigner distribution of Kind-1 of Type-A and Multivariate Inverse -Wigner distribution of Kind-1 of Type-A.

\section{References}

[1] E. Wigner. "On the Distribution of the Roots of Certain Symmetric Matrices." Ann. of Math., 67, 1957, 325-327

[2] Abramowitz, M., and Stegun, I.A. (eds), Handbook of Mathematical Functions, New York: Dover, 1965.

[3] F.A. Berezin Some remarks on the Wigner distribution, Teoret. Mat. Fiz., 17,1973, 305-318

[4] Mardia,"The von Mises Distribution Function," Applied Statistics, 24, 1975, (pp. 268-272)

[5] Geoffrey S. Watson, Distributions on the Circle and Sphere, Journal of Applied Probability Vol. 19, Essays in Statistical Science 1982 pp. $265-280$

[6] Watson, G.S. , Statistics on Spheres, New York: Wiley, 1983.

[7] Z. D. Bai, Y. Q. Yin. Convergence to the Semicircle Law, The Annals of Probability.Volume 16, Number 2 , 1988, 863-875

[8] Fang, K.T., Kotz, S., and Ng, K.W. , Symmetric Multivariate and Related Distributions, London: Chapman and Hall,1990.

[9] Gradshteyn, I.S., and Ryzhik, I.M. , Table of Integrals, Series, and Products, fifth edition edited by A. Jeffrey, San Diego: Academic Press, 1994

[10] L. Accardi, Y. G. Lu . The Wigner semi-circle law in quantum electrodynamics, Communications in Mathematical Physics (19651997). Volume 180, Number 3, 605-632

[11] A. Boutet de Monvel and A. Khorunzhy On the Norm and Eigen value Distribution of Large Random Matrices, Ann. Probability, 27,1999, 913-944

[12] Evans, M., Hastings, N., and Peacock, B "von Mises Distribution." Ch. 41 in Statistical Distributions, 3rd ed. New York. Wiley, 2000.

[13] Hiai, F. and Petz, D The Semicircle Law, Free Random Variables and Entropy. Mathematical Surveys and Monographs 77, American Mathematical Society, Providence, 2000.

[14] Shimizu.K., and Iida, K. ,"Pearson Type VII Distributions on Spheres," Communications in Statistics - Theory and Methods, 31, 2002, 513-526 
[15] M. C Jones, Arthur Pewsey A Family of Distributions on the Circle, Journal of the American Statistical Association. December 1, 2005, 100(472): 1422-1428

[16] Arthur Pewsey, Toby Lewis, M. C. Jones. The wrapped t-family of circular distributions. Australian \& New Zealand Journal of Statistics 49:1, 2007, 79-91

[17] A.R. Soltani and H. Homei , A generalization for two-sided power distributions and adjusted method of moments, Statistics.43, $2009,611-620$

[18] Toshihiro Abe, Arthur Pewsey. Sine-skewed circular distributions. Statistical Papers, 2009.

[19] Arizmendi, O., Barndorff-Nielsen, O. E., and P'erez-Abreu, V ,On free and classical type G-distributions. To appear Braz. J. Probab. Statist, 2010.

[20] Shogo Kato, M. C. Jones. A Family of Distributions on the Circle With Links to Applications Arising From, Möbius Transformation. Journal of the American Statistical Association 105:489, 2010, 249-262

[21] Solairaju, David Sam and Kavita Devi, A New Generalisation of Sam-Solai's Multivariate Laplace distribution, Global Journal of Mathematical sciences: Theory and Practical, Vol.4, Number 1, 2012, pp.63-80.

[22] Solairaju, David Sam and Kavita Dev, A New Generalisation of Sam-Solai's Multivariate-t-distribution, Global Journal of Mathematical sciences: Theory and Practical, Vol.4, Number 1, 2012, pp.81-95.

[23] Solairaju,David Sam and Kavita Devi, A New Generalisation of Sam-Solai’s Multivariate Cauchy distribution of Type-I, Global Journal of Theoretical and applied Mathematical sciences:Vol.2, Number 1, 2012,pp.49-61.

[24] Solairaju, David Sam and KavitaDevi, A New Generalisation of Sam-Solai's Multivariate Cauchy distribution of Type-II, Global Journal of Theoretical and applied Mathematical sciences:Vol.2, Number 1, 2012, pp.63-75

[25] David Sam,Solairaju and Dawood Ali, A New Generalisation of Sam-Solai's Multivariate symmetric Arcsine distribution of Kind-I,ISOR Journal of Mathematics:Vol.2, Issue 3,(May-June 2012),pp.42-48 